\title{
Numerical Analysis of the Cyclic Mechanical Damage of Li-ion Battery Electrode and Experimental Validation
}

\author{
Xuanchen Zhu', Yu Xie ${ }^{2}$, Haofeng Chen ${ }^{1,2, *}$, Weiling Luan ${ }^{2, *}$ \\ ${ }^{1}$ Department of Mechanical \& Aerospace Engineering, University of Strathclyde, G1 1 XJ, UK \\ ${ }^{2}$ Key Laboratory of Pressure Systems and Safety (MOE), School of Mechanical and Power Engineering, East
}

China University of Science and Technology, 200237, China

\begin{abstract}
Evidences have accumulated that the cyclic diffusion-induced stress within lithiation-delithiation process will result in the cyclically evolutive mechanical damage of battery electrode, which adversely affects the mechanical integrity as well as the performance of the Li-ion battery. In this work, the mechanical degradation of electrode under electrochemical-mechanical condition is innovatively evaluated as a fatigue damage process, governed by the interaction between diffusion behaviour and stress generation, and accumulated fatigue damage affected stress-strain response. Structural configuration of a layered electrode plate is modeled in finite element software ABAQUS and a set of user subroutines are developed to implement the proposed fatigue evaluation approach for battery electrode. The constructed approach is proved to be able to simulate multifarious categories of fatigue damage accumulation trends of battery electrode. The strategy to correlate the electrochemistry represented damage with mechanical fatigue damage are proposed. Experimental performance tests are conducted to parameterize the fatigue damage model within the assessment approach for electrode material $\mathrm{LiNi}_{0.5} \mathrm{Mn}_{0.3} \mathrm{Co}_{0.2} \mathrm{O}_{2}$ (NMC532). After parameterization, further circulating chargingdischarging experiments and fatigue damage simulations with respect to different $\mathrm{C}$-rate conditions are carried out to study the applicability of the proposed evaluation model as well as the assumption between electrochemical and mechanical deterioration. It is observed that the electrode surface adhering to electrolyte is more prone to fracture in the cycling operation. The present research work shows that it is available to apply the fatigue damage method to study the gradually mechanical failure of battery electrode under electrochemical-mechanical condition.
\end{abstract}

Keywords: Li-ion battery electrode, diffusion induced stress, fatigue damage, circulating chargingdischarging performance

\section{Introduction}

Lithium ion battery is one of the sought-after technologies for maintaining energy sustainability, and has been widely used in many appliances varying from portable electronics to electric vehicles $[1,2$, $3]$. As a rechargeable energy supply system, the battery operates with the cyclically dynamic process of lithium intercalation into and extraction from solid electrodes. Accompanying with the Li-ion cyclically diffuse in and out the electrodes, the inhomogeneous lithium distribution will lead to a cyclic field of stress known as diffusion induced stress (DIS) [4, 5, 6]. A mass of numerical studies are devoted to simulate the DIS for multifarious configurations of electrode. Weng et al.[7] worked out an analytical model of DIS for nanowire electrode considering various types of operation conditions. $\mathrm{Ji}$ and Guo [8] developed a model to calculate the concentration and diffusion-induced stress in porous electrode with the consideration of Butler-Volmer reaction kinetics and size polydispersity of

\footnotetext{
* Corresponding author.

Email: haofeng.chen@strath.ac.uk (H Chen), luan@ecust.edu.cn (W Luan) Tel: +44(0) 1415482036
} 
electrode particles. Zhang and co-workers [9] employed both finite difference method and finite element approach to simulate the diffusion induced stress within ellipsoidal electrode particles. Cui and co-workers [10] derived a stress-dependent chemical potential applied to solid-state diffusion. The obtained chemical potential is employed to study the DIS in a spherical Silicon particle under both elastic and elastic-plastic conditions. In reference [11], the phase transitions and lithiation induced stress are investigated for NMC three-dimensional microstructures by employing the finite element method. Zhang et al. [12] have analytically formulated the diffusion induced stress in the layered Liion battery electrode plates, and also discussed the effects of different charging conditions on the DIS. Liu [13] has employed the finite element model to investigate the DIS of a Si thin film bonded to a rigid substrate, where the model incorporates plasticity, interaction between solute diffusion and stress formation, diffusion from the edge surface, and concentration affected material parameters. It can be seen that the lithiation behaviour caused stress is significant to various structures of battery electrode and is required to be evaluated.

Evidences have indicated that the diffusion induced stress will lead to the mechanical destruction which is generally regarded as one of major contributors affecting the electrochemical performance of battery $[4,14]$ and has been intensively studied by means of multifarious methods. Based on the linear elastic fracture mechanics, $\mathrm{Hu}$ [15] described an approach to determine the critical condition for crystalline particle to avoid insertion induced cracking. Within the framework of $J$ integral in elasticplastic fracture mechanics, Chew [16] demonstrated the in-plane cracking of silicon thin film electrode during lithiation and delithiation. By means of phase field method, Klinsmann and co-workers [17, 18] presented a coupling model incorporating diffusion, stress and crack growth to study the effects of particle size, initial crack and flux on the crack formation. By using discrete element method associating with the cohesive crack model, Sun et al. [19] investigated the crack initiation and propagation behaviour at interfaces between the primary particles in secondary cathode particle. $\mathrm{Hu}$ et al. [20] employed the stress intensity factor coupled with surface effect to study the crack growth in nano-spherical electrodes. Li and co-workers [21] applied the spring-block model to investigate the cracking behaviour of silicon thin film electrode, and obtained a critical thickness for avoiding crack of silicon film. By using in-situ crack monitoring, ex-situ Li concentration measurement, and finite element simulation, Yang et al. [22] observed the Li-ion redistribution around crack tip caused by coupled diffusion-stress mechanism for battery electrode. In our preliminary research [23], we also developed finite element subroutines to perform the coupled diffusion-stress analysis and employed the extended finite element method to study the intragranular fracture behaviour within primary particle under DIS. These research works have investigated the cracking behaviour for battery electrode under electrochemical condition and demonstrated the importance of studying the mechanical failure of battery electrode under diffusion-induced stress.

With the consideration of practical operation of battery electrode, the degradation can be regarded as a progressive process with the proceeding of cyclic operation. Chen et al. [24] employed SEM to investigate the mechanical damage in $\mathrm{LiMn}_{1.95} \mathrm{Al}_{0.05} \mathrm{O}_{4}$ electrode. The research work presented that, under electrochemical cycling, the failure in electrode material is a fatigue process instead of the commonly assumed brittle fracture. Besides, many other research works have also presented the effect of fatigue behaviour on Li-ion battery in recent years. In reference [25], the fatigue induced spatial lithium inhomogeneities along the radial direction of a cylinder-type Li-ion cell is investigated. Schweidler et al. [26] presented a work on the long-term cycling performance of graphite/NCM851005 cell. They specifically associated the electrochemical results with (micro)structural data from operando X-ray diffraction and electron microscopy to study the fatigue behaviour. By employing in situ neutron powder diffraction and electrochemical impedance spectroscopy [27], the fatigue 
process is investigated as a function of cycling temperature for different types of Li-ion batteries. Nevertheless, how to quantitatively evaluate the fatigue induced degradation under electrochemicalmechanical condition still remains to be clarified.

In this work, we propose that the continuum damage mechanics founded by Kachanov [28] can be applied to quantify the fatigue caused damage of Li-ion battery under electrochemical cycling. Building on the continuum mechanics and thermodynamics, the damage mechanics theory aims to derive the continuous evolution equation of damaged material by defining the applicable damage variable, so as to describe the dynamic stress-strain-damage field of the target solid [29]. The continuum damage mechanics has been adopted in the assessment of damage phenomenon like elasticity, plasticity, viscoplasticity, creep, fatigue, etc., and is available to describe the damage for metallic material, composite material, concrete material and so on [30, 31, 32, 33]. Employing the continuum damage mechanics in studying the mechanical failure behaviour of electrode is accessible to accurately track the overall degradation process of electrode during circulating operation.

This paper takes the lead to model the continuous mechanical degradation of battery electrode under diffusion-induced stress as a fatigue damage process. The proposed fatigue evaluation approach for battery electrode is built on the continuum damage mechanics, and takes into consideration the coupling effect between diffusion behaviour, diffusion induced stress formation, and accumulated fatigue damage affected stress-strain response. Relevance assumption is made to connect the electrochemistry represented damage with mechanical fatigue damage. Circulating chargingdischarging test cycled at the $\mathrm{C}$-rates of $1 \mathrm{C}$ condition is performed as a benchmark to fit the fatigue parameters in the proposed cumulative damage model. The established approach is then applied to simulate other multi-electrochemical conditions and further studied by comparing these numerical simulations with conducted experimental tests under the same multi-electrochemical conditions. This research work presents that it is accessible to quantify the fatigue degradation of Li-ion battery electrode based on the continuum damage mechanics and provides understanding for mechanical failure of Li-ion battery electrode.

\section{Theoretical model}

\subsection{Coupled diffusion-stress problem}

Consider a diffusion process which obeys the following mass conservation law:

$$
\frac{\partial c}{\partial \tau}+\nabla J=0
$$

where $c$ refers to the solute concentration, and $\tau$ is defined as time. $J$ denotes the diffusion flux which measures the amount of substance what will flow through a unit area during a unit time interval. One can make a heat conduction process as the comparison, which satisfies the following energy conservation law:

$$
\rho c_{s} \frac{\partial t}{\partial \tau}+\nabla q=\varnothing
$$

Where $\rho, c_{s}, t, \emptyset$ represent density, specific heat, temperature, and internal heat source respectively. $q$ denotes the heat flux, which describes the flow of energy per unit of area per unit of time. According to the hypothesis as follows, mass diffusion behaviour could be analogized as heat conduction behaviour [34].

$$
\begin{array}{ll}
t=c & \emptyset=0 \\
q=J & \rho c_{s}=1
\end{array}
$$


The diffusion flux $J$ in formula can be defined based on the Fick diffusion law so as to take into account the bidirectional effect between Li-ion diffusion and stress generation [35],

$$
\begin{gathered}
J=-D_{f} \nabla c \\
D_{f}=D_{f 0} \exp \left(\frac{\Omega \sigma_{h}}{R T}\right)
\end{gathered}
$$

where $D_{f}$ is the diffusion coefficient, and $D_{f 0}$ is the diffusion coefficient at stress-free condition. $\Omega$ refers to the partial molar volume of Li-ion. $R$ is a gas constant, $T$ is absolute temperature. $\sigma_{\mathrm{h}}$ is the hydrostatic stress. The stress effect on diffusion behaviour can then be incorporated in calculating diffusivity and diffusion flux in every iteration step. By developing a set of UMATHT subroutine [36], we implement the aforementioned formulas in ABAQUS to perform coupled diffusion-stress evaluation. As shown in Appendix A, the UMATHT subroutine first calculates the hydrostatic stress for current integration point, and then calculates the diffusivity and diffusion flux with the consideration of stress effect to update the corresponding parameters in transient heat conduction equation.

In situations when the plastic deformation is considered, an elastic-perfectly plastic constitutive model is adopted to describe the material behaviour. According to the analogy between DIS and thermal stress $[9,35]$, the increment of the total strain $d \varepsilon_{i j}$ can be written as

$$
d \varepsilon_{i j}=d \varepsilon_{i j, e}+d \varepsilon_{i j, p}+d \varepsilon_{i j, c}
$$

where $d \varepsilon_{i j, e}$ is the increment of elastic strain, and follows Hooke's law

$$
d \varepsilon_{i j, e}=\left[(1+v) d \sigma_{i j}-v d \sigma_{k k} \delta_{i j}\right] / E
$$

$E, v$ and $\delta_{i j}$ represent elasticity modulus, Poisson's ratio, and kronecher symbol respectively. $d \varepsilon_{i j, p}$ is the plastic part and obeys $J_{2}$-flow rule

$$
d \varepsilon_{i j, p}= \begin{cases}0, & \sigma_{e q}<\sigma_{s} \\ 0, & \sigma_{e q}=\sigma_{s}, \quad d \sigma_{e q}<d \sigma_{s} \\ \lambda s_{i j}, & \sigma_{e q}=\sigma_{s}, \quad d \sigma_{e q}=d \sigma_{s}\end{cases}
$$

where $s_{i j}=\sigma_{i j}-\frac{\sigma_{k k} \delta_{i j}}{3}$ is the deviatoric stress, $\sigma_{e q}=\sqrt{3 s_{i j} s_{i j} / 2}$ is the equivalent stress, and $\sigma_{s}$ is yield strength. In perfectly plastic model, $\lambda$ is a scalar that can be determined by the boundary-value problem. $d \varepsilon_{i j, c}$ is the lithiation-caused strain and can be determined by [9, 34]

$$
d \varepsilon_{i j, c}=\frac{\Omega}{3} d c \delta_{i j}
$$

\subsection{Mechanics and experiment represented damage models}

In practical operation, the continuously mechanical degradation on electrode accompanies the capacity fading of battery. One can make the assumption that, the mechanical damage over cycles is in proportion to the capacity fading represented electrochemical-damage. Based on the continuum damage mechanics (CDM), we propose a cumulative damage model to describe the deterioration of electrode in mechanical way. In addition, through performing charge-discharge tests, an experimental index of damage can also be defined so as to correlate with the mechanical damage.

\subsubsection{Continuum damage mechanics}

The research objective of continuum damage mechanics is the deformed solid containing multifarious categories of microdefects, which are regarded to continuously distribute in the target solid [29]. Under the action of external factors, the continuous nucleation, propagation and aggregation of 
microdefects will lead to the degradation of material and structure. This type of continuously distributed microdefects can be depicted by a field variable known as damage field. If the distribution of damage and its effect on the material performance have no directional difference, the damage state can be regarded as isotropic and expressed by employing a scalar damage variable $\mathrm{D}(0 \leq \mathrm{D} \leq 1)$. In the present work, the symbol D makes a general reference to various types of damage field including the calculated mechanical damage and the experiment indicated damage.

The evolution of microdefects or the growth of damage can reduce the load-carrying area, which will further magnify the stress. It is then available to obtain the following relation

$$
\tilde{\sigma}=\sigma \frac{d A}{d A-d A_{D}}
$$

where $d A$ denotes the area before damage, and $d A_{D}$ is the detracted area caused by damage. $\tilde{\sigma}$ is defined as effective stress. Based on the definition of damage, one obtains [29]

$$
\tilde{\sigma}=\frac{\sigma}{1-D}
$$

According to the strain equivalence principle, the strain caused by the effective stress $\tilde{\sigma}$ in the fictitious undamaged material is in equivalence to the strain of damaged material under nominal stress $\sigma$. The constitutive relation of the damaged material can thereby be derived from that of the undamaged material by using the effective stress of damaged material to replace the nominal stress in the constitutive relation of undamaged material.

$$
\varepsilon=\frac{\widetilde{\sigma}}{E_{0}}=\frac{\sigma}{E(D)}
$$

where $E_{0}$ and $E(D)$ represent the Young's modulus of the fictitious undamaged material and that of the damaged material. Combining Eq. 11 and 12, one obtains

$$
(1-D) E_{0}=E(D)
$$

It is worth reminding that, the $E(D)$ is the factitiously revised Young's modulus for reflecting the effect of damage on material stress-strain response.

\subsubsection{Fatigue damage model}

In this work, we regard the mechanical deterioration of electrode as a fatigue damage course. The cycling number of charge-discharge tests indicate that it is a strain-controlled fatigue failure, where the number is normally less than $10^{5}$. Manson and Coffin [37] postulated that the fatigue life can be predicted by employing plastic strain amplitude as the input parameter.

$$
\frac{\Delta \varepsilon_{p}}{2}=\varepsilon_{f}^{\prime}(2 N)^{c^{\prime}}
$$

where $\frac{\Delta \varepsilon_{p}}{2}$ is the plastic strain amplitude, $2 N$ is the number of reversals to failure. $\varepsilon_{f}$ and $c^{\prime}$ are two empirical constants known as the fatigue ductility coefficient and the fatigue ductility exponent respectively. By formula derivation, it is accessible to obtain the following expression, which can be applied to predict the mechanical damage per cycle $d D_{m i}$ with specific plastic strain range.

$$
2\left(\frac{2 \varepsilon_{f}^{\prime}}{\Delta \varepsilon_{p}}\right)^{\frac{1}{c^{\prime}}}=\frac{1}{N_{i}}=d D_{m i}
$$

And the accumulated mechanical damage $D_{m}$ over cycles is given by 


$$
D_{m}=\sum_{i=1}^{n} d D_{m i}
$$

This accumulated fatigue damage varies at each material point which can be regarded as integration point in finite element analysis. Additionally, the damage also affects the yielding stress for the damaged material [38]. It is worth emphasizing that, the revised physical properties, such as Young's modulus and yield strength, are factitiously equivalent conversions in the continuum damage mechanics for representing the degradation behaviour of the damaged material. The following expression is proposed to consider the effect of damage on yield strength.

$$
\sigma_{s 0}(1-D)^{n_{r}}=\sigma_{s}(D)
$$

where $\sigma_{s 0}$ and $\sigma_{s}(D)$ denote the yield strength of initial undamaged material and damaged material, respectively. $n_{r}$ is a nonlinearity index which determines the damage accumulation trend. And the value of $n_{r}$ allows to reflect the different effect degrees of damage on Young's modulus and yield stress. From a physical point of view, this index is set to be greater than or equal to $0\left(n_{r} \geq 0\right)$, as the damage is considered to reduce the yield strength or have no impact on it under special circumstances. An UMAT subroutine is developed to simulate the stress-strain response with the consideration of damage effect, as shown in Appendix A. It is presented that, the accumulated damage is first applied to factitiously revise the Young's modulus and yield strength of the material. Then the elastic-perfectly-plastic constitutive equation considering diffusion strain is used to describe the material response. Based on the Manson-coffin model, the maximum plastic strain range in the lithiation-delithiation process is employed to calculate the damage generated in one cycle.

With the developed UMAT-UMATHT subroutines, we conduct the coupled diffusion-stress analysis considering fatigue damage and exhibit the effect of $n_{r}$ on damage evolution. Fig 1 presents three categories of damage evolution trends with respect to three representative values of $n_{r}$, where the rest material properties are set as constant in these three scenarios. The damage produced from one cycle will be less than that of the subsequent cycle when $n_{r}>1$ (15 as example) is satisfied. The damage then accumulates in a progressive way and changes the yield stress greatly than the Young's modulus. The damage accumulation will continue in a linear way when $n_{r}=1$, where the damage produced from two neighbouring cycles are equivalent, and affects the yield strength and Young's modulus to the same degree. In the third scenario, $0 \leq n_{r}<1$ (0.001 as example), the damage generated from one cycle is slightly greater than that of the subsequent cycle leading to the slight degressive accumulation of damage. And the damage effect on yield stress is less than that of Young's modulus. Notably, the proposed damage model is capable of simulating multifarious types of damage accumulation rules. Corresponding to experimental results and relevance assumptions, one can fit $n_{r}$ so as to correlate the modeled damage with experimental damage.

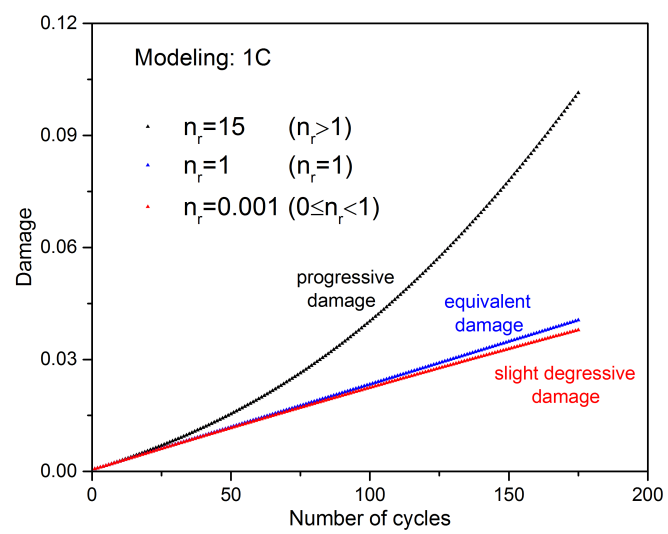

Fig 1. The modelled three categories of damage evolution trends with respect to different values of 


\subsubsection{Capacity fading represented damage rule}

In a broad sense, one measurable state variable which monotonically changes with the proceeding of cyclic course, can be employed as the measurement of fatigue damage. For battery electrode, the continuous mechanical fatigue degradation is simultaneous with the capacity fading process of battery $[4,14]$. Therefore, we select the capacity as an experimental index to compare with the successive mechanical fatigue damage. Considering the following expression

$$
C_{e}=C_{e 0}\left(1-D_{e}\right)
$$

where $C_{e 0}$ denotes the battery capacity before cycling and $D_{e}$ is the cyclic charge-discharge experiment indicated damage. $C_{e}$ is battery capacity corresponding to damage $D_{e}$ after a certain number of lithiation-delithiation cycles. The experiment indicated damage and battery capacity hold the following relationship.

$$
D_{e}=1-\frac{C_{e}}{C_{e 0}}
$$

Through cyclic charge-discharge tests, one can quantificationally obtain the capacity determined damage.

\section{Finite element model}

In this work, we apply the finite element model to evaluate the mechanical fatigue deterioration of NMC532 layered electrode with developing a set of UMAT-UMATHT subroutines, which comprehensively take damage accumulation, interaction between stress and diffusion, damage affected stress-strain response into consideration. In performed experiments, the cathode active layer is a thin film with $12 \mathrm{~mm}$ diameter and 20um thickness as shown in Fig 2 (a). For studying the evolution of fatigue damage along the direction of thickness, the finite element model of cathode active layer is built as a disk with 300um diameter and 20um thickness as presented in Fig 2 (b), where the diameter size is set as 15 times bigger than thickness size so as to reflect the structural character of thin film and make it available to present the fatigue damage distribution along the thickness. According to the analogy assumption in section 2.1, the coupled temperature-displacement element is selected and associated with the developed UMATHT subroutine to perform the coupled diffusion-stress analysis by employing the coupled temperature-displacement modulus in ABAQUS. Considering the computational task load for simulating more than 150 lithiation-delithiation cycles, the 20 nodes reduced integration element type is picked to ensure the accuracy and efficiency of the calculation. Hence, C3D20RT element (20 nodes thermal cubic elements with reduced integration) is adopted for conducting coupled diffusion-stress-damage analysis of electrode layer. The top surface of active layer adheres to electrolyte, and serves as the access for lithiation and delithiation. The diffusion boundary condition herein is given by the Neumann boundary condition

$$
J=\frac{i_{n}}{F_{a}}
$$

where $i_{n}$ is the current density applied on the top surface, $F_{a}$ is Faraday constant. The bottom surface of active layer is bonded to a rigid substrate made of Al. As the Young's modulus of current collector is far larger than that of the active layer [39], the current collector is thereby considered as a rigid surface in this work. 


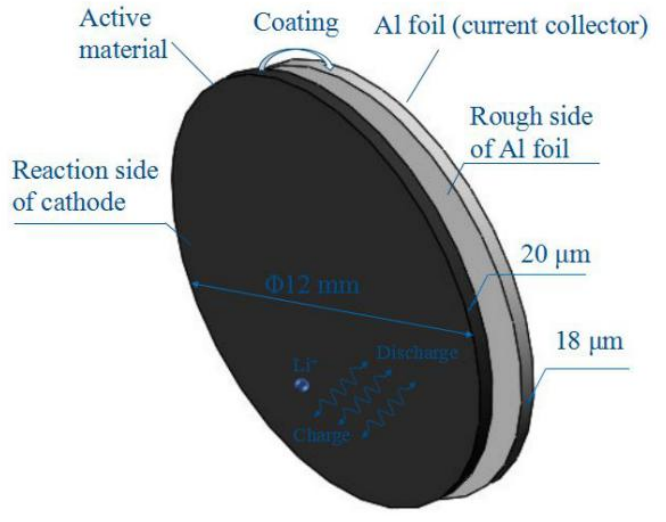

(a)

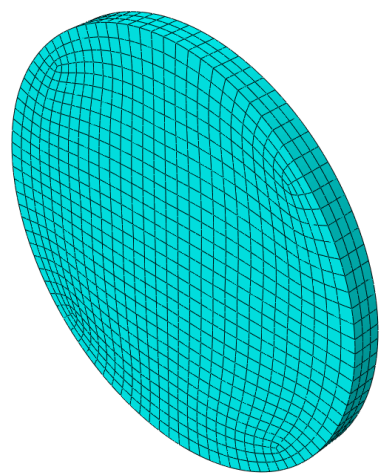

(b)

Fig 2 (a) Schematic diagram of the experimentally tested layered electrode and current collector, (b) mesh of the cathode active layer

The material properties adopted in this numerical work are listed in Table 1. The NMC cathode is assumed as an elastic-perfectly plastic material, which experiences no work-hardening during plastic deformation. The elastic-perfectly plastic model is widely used in the simulation of mechanical behaviour for electrode material $[43,44]$, as the hardening behaviour is not significant for such a material and it is available to model the stress-strain response of the material in the lack of hardening material properties. The yield strength of NMC electrode material is in a range, and is decided by material preparation, material composition, etc. It cannot be measured accurately by the experiment due to the preparation technology of oxide electrode material, and there is no accurate value of yield strength for NMC material in the literature. The commonly used yield strength of oxide electrode material having similar lattice structure with NMC is applied in this work, and is assumed as $100 \mathrm{MPa}$. Besides, there is no reliable experimental data on the fatigue ductility coefficient and the fatigue ductility exponent for NMC electrode material, which are only available for the common structural materials. Therefore, based on the relevance assumption that the fatigue damage is in proportion to the experiment represented damage, we use capacity variation from the electrochemical performance test at $1 \mathrm{C}$ as the benchmark to fit the fatigue parameters. After the parameterization, the proposed model is then available to simulate mechanical degradation of electrode under other electrochemical conditions. Experimental performance tests under the same electrochemical conditions are further conducted so as to verify the obtained parameterized fatigue damage model and relevance assumption via comparing the mechanical fatigue damage with electrochemistry indicated damage.

Table 1. Material properties

\begin{tabular}{ll} 
Property & Value \\
\hline Young's modulus at undamaged status (GPa) & $6.5^{[39]}$ \\
Poisson ratio & $0.3^{[40]}$ \\
Diffusivity $\left(\mathrm{cm}^{2} / \mathrm{s}\right)$ & $10^{-11[41]}$ \\
Partial molar volume $\left(\mathrm{m}^{3} / \mathrm{mol}\right)$ & $2.1 \mathrm{E}-6^{[42]}$ \\
Yield strength $(\mathrm{MPa})$ & $100^{-140}$ \\
\hline
\end{tabular}




\section{Experimental test}

\subsection{Electrode preparation}

The preparation of NMC532 cathode consists of the following steps: the NMC532 powder (commercial supplied) and acetylene are added in a mortar with a mass ratio of 8:1, and then they are ground to form a homogeneous mixture. The adopted mass ratio is an empirical ratio which is commonly used in the preparation of electrode $[45,46]$, and the performance of electrode with this ratio is relatively stable. NMP-based suspension is prepared by dispersing polyvinylidene fluoride binder (PVDF) in Nmethyl-2-pyrrolidone, and the weight of PVDF is the same as acetylene in the obtained mixture. The NMC532 slurry is then produced by dispersing the obtained powder mixture in the prepared suspension for $6 \mathrm{~h}$ at $800 \mathrm{RPM}$. Subsequently, the slurry is coated on the current collector, Al foil, with a density of $3.7 \mathrm{mg} / \mathrm{cm}^{2}$, then the obtained slice is dried at $120 \mathrm{C}^{\circ}$ in electric vacuum drying oven for $12 \mathrm{~h}$ to remove the solvent. The prepared cathode is trimmed in round slice with a diameter of $12 \mathrm{~mm}$ as working electrode. The areal weight of the NMC532 material is about $2.7 \mathrm{mg} / \mathrm{cm}^{2}$ and the composition of active layer is shown in Table 2. All working electrodes are then dried at $60 \mathrm{C}^{\circ}$ in electric vacuum drying oven for $6 \mathrm{~h}$ to remove moisture before they are transferred into a glovebox.

Table 2. The composition of active layer

\begin{tabular}{ll} 
Ingredient & Mass percent \\
\hline NMC532 powder & $80 \%$ \\
Acetylene & $10 \%$ \\
Polyvinylidene fluoride & $10 \%$ \\
\hline
\end{tabular}

\subsection{Cell assembling}

The electrochemical performance of the cathode is assessed with assembling CR2025-type coin cells. The prepared working electrode is employed as the cathode. A $1 \mathrm{M}$ solution of LiPF6 dissolved in EC: DEC (volume ratio=1:1) is used as the electrolyte, and lithium metal is employed as the anode. The separator of cell is polypropylene membrane (Celgard 2500). The CR2025-type coin cells are assembled in a glove box under a dry argon atmosphere (moisture and oxygen less than $0.1 \mathrm{ppm}$ ). To ensure a good contact between components of cells, all cells are placed for $12 \mathrm{~h}$ before test.

\subsection{Electrochemical performance measurement}

The electrochemical performance is investigated by using Land Cell System (CT2001A, Wuhan Jinnuo Electronic Co. Ltd, Wuhan China). The cells are cycled between 2.8 and $4.3 \mathrm{~V}$ vs Li/Li+ via VSP potentiostats at different rates under galvanostatic condition. As an activation process, all cells operate for one cycle at the C-rates of $0.1 \mathrm{C}, 0.2 \mathrm{C}$ and $0.5 \mathrm{C}$ in turn. Subsequently, cells are cycled at the targeted C-rate of $0.5 \mathrm{C} / 1 \mathrm{C} / 2 \mathrm{C}$ until the capacity decreases to 80 percent. Where 80 percent of the initial capacity is the typical criteria for electric vehicle applications [47, 48]. Hereafter, the discharge capacity and the performance of capacitor can not meet the needs of use very well. Four samples are tested under each C-rate condition and the average capacity fading is used to define the experiment indicated damage. Fig 3 exhibits the schematic diagram of electrochemical performance measurement and the electrochemical performance of coin cell at cycling window of 2.8-4.3 V under $1 C$. 


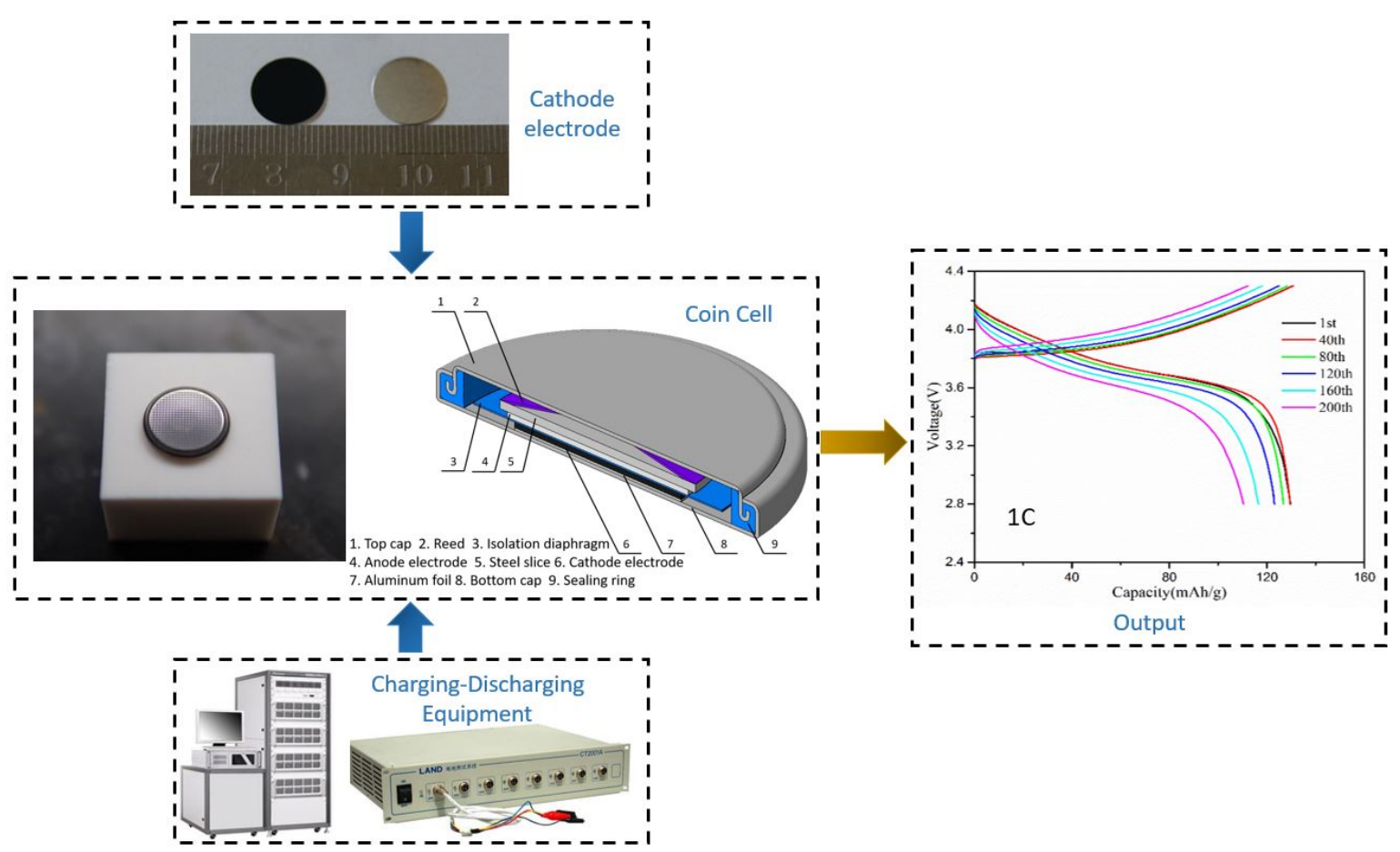

Fig 3 Schematic diagram of electrochemical performance measurement and experimental output

\section{Results and discussion}

\subsection{Model establishment and validation under various C-rate}

From electrochemical performance measurements, we acquire the degradation trend of discharge capacity with respect to the number of cycles for coin cell under $1 \mathrm{C}$ and plot it in Fig 4 . The irregularity of polarization voltage inside the battery and test accuracy range of the equipment result in the slight rebound of capacity in a few neighbouring cycles. It is common and inevitable in the chargingdischarging test $[49,50,51]$, but will not influence the overall variation trend of the capacity. It is available to quantify this degradation by defining a capacity fading represented damage via the aforementioned expression in Eq. (19). The evolution trend of this capacity data decided damage is also presented in Fig 4 . The result indicates that the capacity presents a fluctuated downtrend with the proceeding of cycle process. The corresponding damage thereby follows an uptrend and almost obeys a linear dependence with the number of cycles. Hence the effect index $n_{r}$ in cumulative damage model can be set as 1 for this case. What can also be observed is that it takes around 82 cycles and 157 cycles for experiment defined damage to accumulate to 0.1 and 0.2 at $1 \mathrm{C}$. The cell is cycled until failure when it reaches $80 \%$ of the initial capacity, which is the typical standard for the cell. Hence, the corresponding damage limit is around 0.2 . 


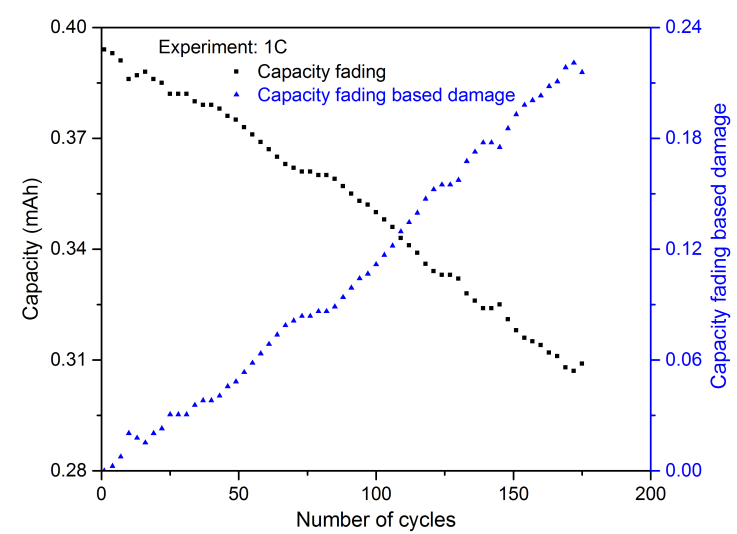

Fig 4. The variation of capacity and capacity fading represented damage with respect to the number of cycles for coin cell under $1 \mathrm{C}$

For the layered electrode model shown in Fig. 2, one can make the assumption that the fatigue damage of top surface adhering to electrolyte is in equivalence to the proposed capacity fading represented damage. As the mechanical collapse caused by fatigue damage on the top surface will interfere the channel for Li-ion transportation and interdict the electrochemical process. The fatigue damage on the top surface thereby dominates the overall mechanical damage status of the active layer. In this work, we first calculate the history of plastic strain range for NMC532 electrode plate cycled at $1 \mathrm{C}$ without considering fatigue damage effect, and the obtained plastic strain range at steady state is $\mathbf{0 . 0 4}$. According to the aforementioned analogous assumption, it is accessible to fit the initial fatigue parameters in computational equation (15) on mechanical damage from this plastic strain range by comparing with the capacity fading represented damage in Fig. 4. With employing the fitted initial fatigue parameters, we can calculate the history of plastic strain range of electrode plate at $1 \mathrm{C}$ for the second time and the fatigue damage can also be given. The newly obtained plastic strain range is 0.0392 and the calculated fatigue damage is relatively less than the experimental damage via utilizing the proposed relevance assumption. Hence, we fit the fatigue parameters based on the current plastic strain range again and the newly obtained fatigue parameters are employed to conduct the next calculation. Working as an iterative process, the correlation coefficient between the calculated fatigue damage and experiment indicated damage reaches 0.9956 in the fourth calculation, and the current fatigue properties are regarded as the finial values for NMC532 active material. Subsequently, we apply the parameterized fatigue damage model to predict the mechanical degradation of electrode under other $\mathrm{C}$-rates $(0.5 \mathrm{C}, 2 \mathrm{C})$ and compare with the experiment indicated damage to study the rationality of the fatigue damage model and relevance assumption. The fitted material properties are summarized in Table 3 , where $\varepsilon_{f}^{\prime}$ and $c^{\prime}$ denote the fatigue ductile coefficient and fatigue ductile exponent respectively.

Table 3. Fitted parameters in the fatigue damage model

\begin{tabular}{ll}
$\varepsilon_{f}^{\prime}$ & $c^{\prime}$ \\
\hline 3.184 & -0.688 \\
\hline
\end{tabular}

We show below in Fig. 5 (a) the fatigue damage contour of electrode under $1 \mathrm{C}$, and it is suggested that the produced mechanical damage is layered distributed along the direction of Li-ion flow. Fig 5 (b) depicts the fatigue damage distribution along the direction of thickness after 50, 100 and 150 cycles. It is observed as a general trend that the fatigue damage of top surface adhering to electrolyte far exceeds that of the rest region, which indicates the top surface of electrode is more prone to fracture. Fig. 6 presents the evolution trends of calculated fatigue damage and experiment represented damage 
cycle by cycle under $1 \mathrm{C}$, where integration point 1 is on the top surface adhering to electrolyte and integration point 2 is on the bottom surface bonded to the rigid substrate.

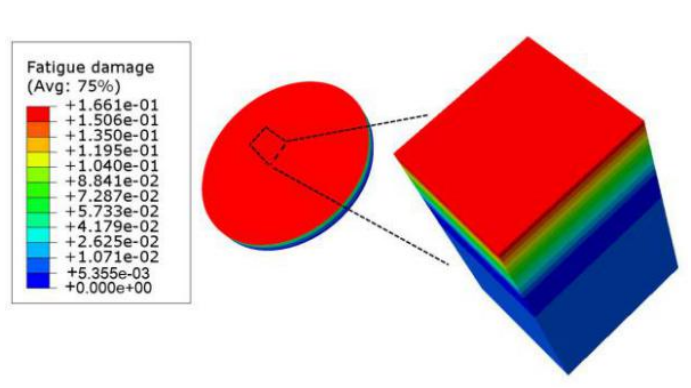

(a)

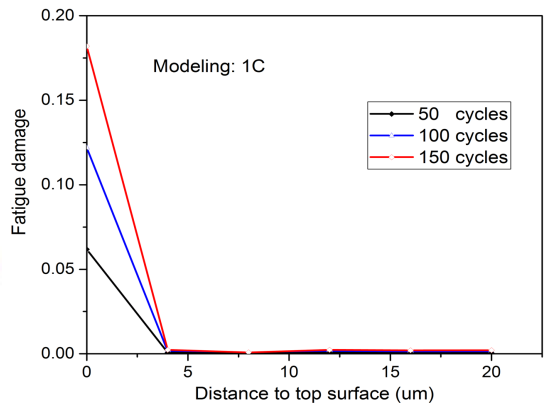

(b)

Fig. 5 (a) Contour plot of damage distribution on the layered electrode at 1C, (b) fatigue damage distribution along the direction of thickness

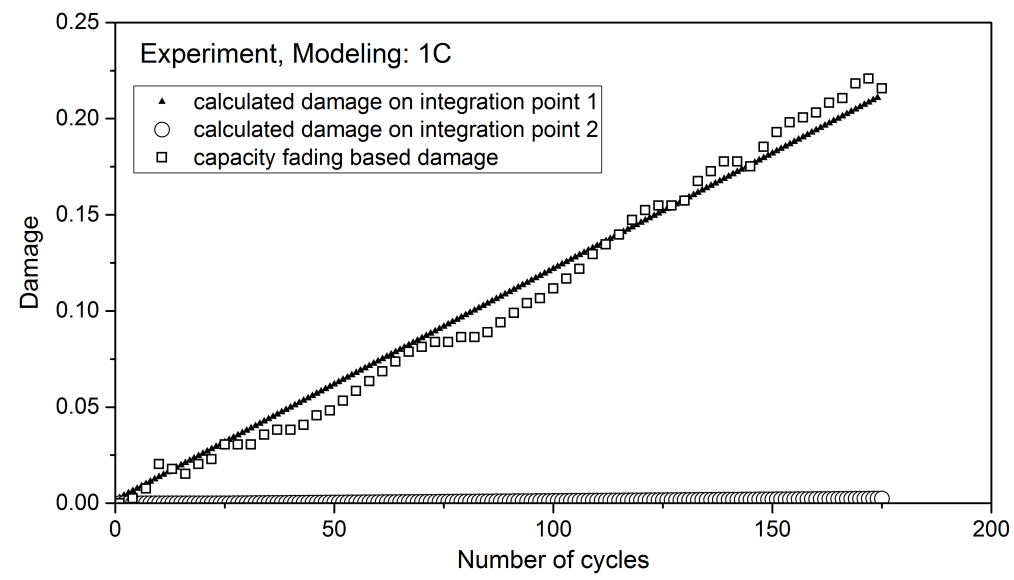

Fig 6 Capacity fading represented damage, calculated fatigue damage on integration point 1 and 2 against the number of cycles under $1 \mathrm{C}$

By performing further electrochemical performance tests, it is available to obtain the degradation trends of capacity and determine the evolution of capacity fading based damage under other C-rate conditions $(0.5 \mathrm{C}$ and $2 \mathrm{C})$. The mechanical damage of electrode has been simulated by applying the proposed cumulative damage model with the same electrochemical loads as the tests conducted experimentally. According to the presented relevance assumption, the parameterized fatigue damage model is validated herein by comparing the calculated damage results with the experiment indicated damage.

The experimental results of electrochemical performance tests cycled at $0.5 \mathrm{C}$ and $2 \mathrm{C}$ are presented in Fig 7 (a) and (b). Based on the definition in Eq. 19, the capacity data is extracted to decide the experimental damage also shown in Fig 7 (a) and (b), where the damage presents a linearly fluctuated uptrend over cycles in both scenarios. It takes around 98 cycles and 85 cycles at $0.5 \mathrm{C}$ and $2 \mathrm{C}$ to reach 0.1 of damage respectively. It can be found that, the capacity fading under low C-rate $(0.5 \mathrm{C})$ is relatively slower than the degradation under high $\mathrm{C}$-rates $(1 \mathrm{C}, 2 \mathrm{C})$. 


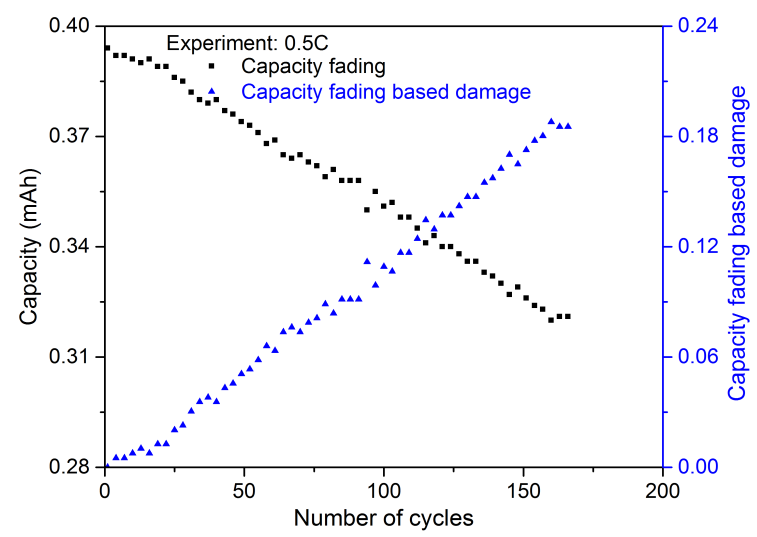

(a)

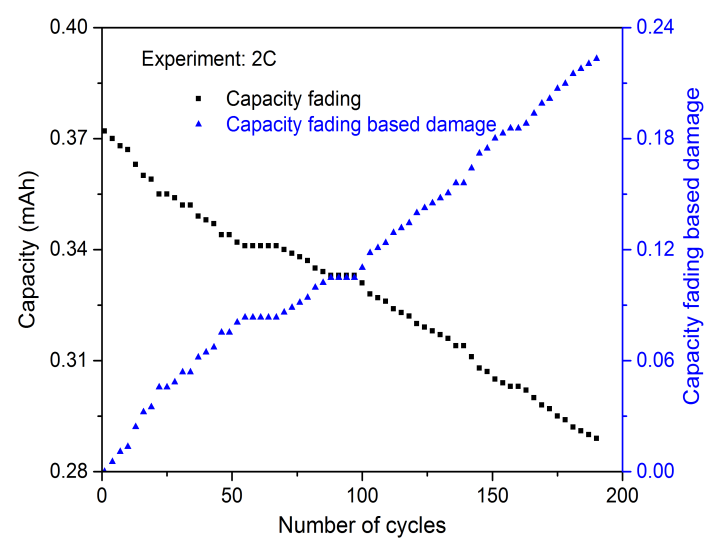

(b)

Fig 7. The variation of capacity and capacity fading represented damage with respect to the number of cycles for coin cell under (a) 0.5C, (b) $2 \mathrm{C}$

The parameterized fatigue damage model is adopted to track the mechanical damage evolution of lithium battery electrode cycled at $0.5 \mathrm{C}$ and $2 \mathrm{C}$. Fig 8 (a) and (b) depict the evolution trends of capacity fading represented damage and calculated fatigue damage on integration point 1,2 at $0.5 \mathrm{C}$ and $2 \mathrm{C}$. It is found that the calculated damage under these two scenarios both follow an approximately linear dependence with the number of cycles. The comparison between the damage on integration point 1 and the experiment indicated damage suggests that, under the researched electrochemical condition, it is accessible to employ the continuum damage approach to track the mechanical degradation of electrode.

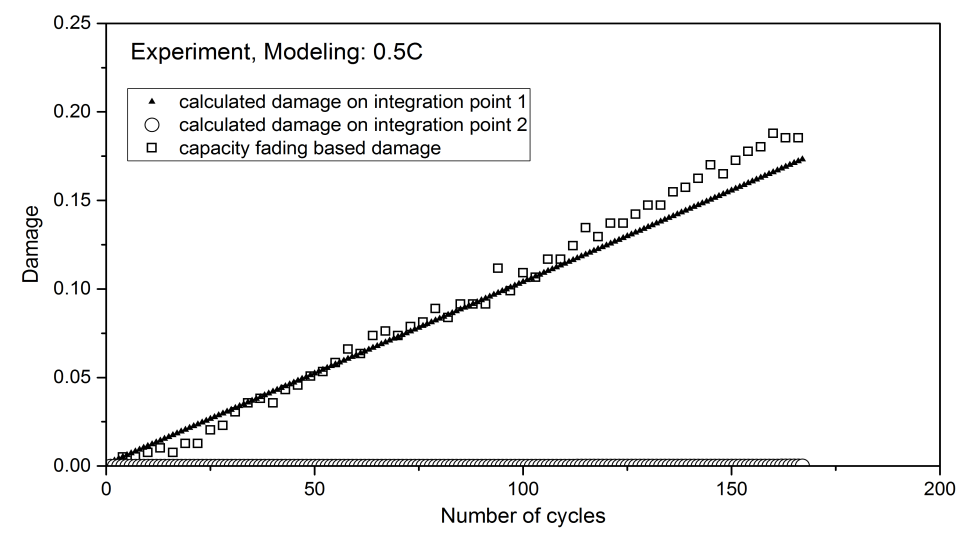

(a)

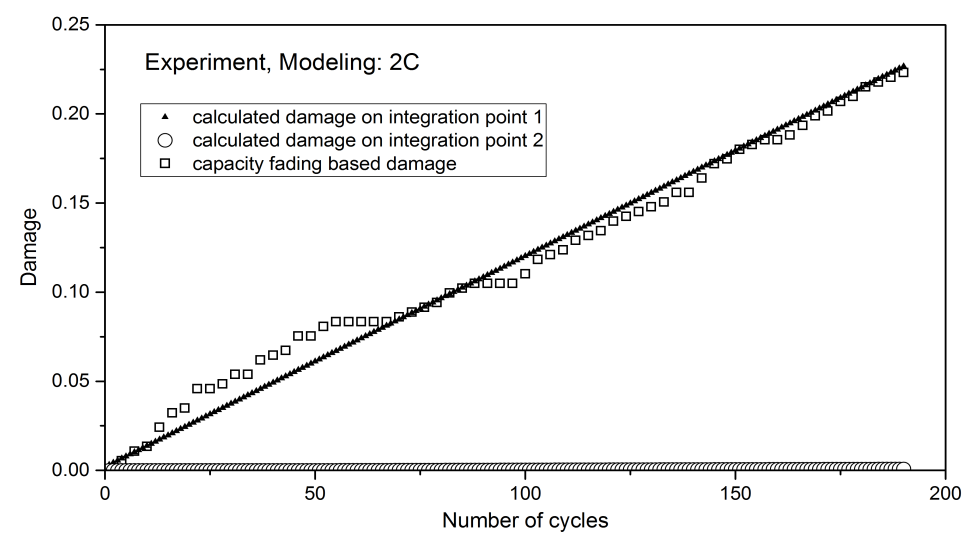

(b)

Fig 8. Capacity fading based damage, calculated damage on integration point 1 and 2 against the number of cycles under (a) $0.5 \mathrm{C}$, (b) $2 \mathrm{C}$ 


\subsection{Damage affected stress-strain response}

According to the description in section 2.2, the fatigue damage generated from one cycle can be incorporated in factitiously modifying the Young's modulus and yield strength, which makes the material more prone to deform. Fig 9 (a) depicts the monotonic reduction of the revised Young's modulus and yield strength for NMC532 cathode with the proceeding of cycles on two integration points at $1 \mathrm{C}$. As the fatigue damage accumulation rate of integration point 1 adjacent to electrolyte is considerably faster than integration 2 bonded to current collector, the descent rate of Young's modulus and yield strength for top surface of electrode is more rapid than that of the opposite. Besides, Fig 9 (b) shows the cross-sectional view of the deformed active layer after 150 cycles, where the meshes represent the initially undeformed active layer. It is observed that, after 150 cycles, the overall structure shape is greatly changed due to the lithiation behaviour. The corresponding concentration distribution within active layer is given in Fig 9 (c) and the residual concentration gradient caused by Li-ion trapping mechanism results in the expansion phenomenon of the active layer. Fig 10 (a) and (b) present the distribution of in-plane stress component and von Mises stress on electrode thickness section at the end of lithiation phase of $50^{\text {th }}$ and $150^{\text {th }}$ cycle under $1 \mathrm{C}$ respectively. The structure character of electrode plate decides the fact that two in-plane stress components are equivalent and much larger than the stress component along the thickness direction. The distribution of in-plane stress component shows that the electrode plate is in compressive status at the end of lithiation phase. By comparing the von Mises stress with damage modified yield strength, it is observed that the upper layer adjacent to electrolyte and the lower layer closing to current collector are in a state of plastic yielding at this moment. Due to the rapid rise of fatigue damage, the stress field of upper layer is significantly affected, while the stress of lower layer is hardly changed as little fatigue damage is generated here.

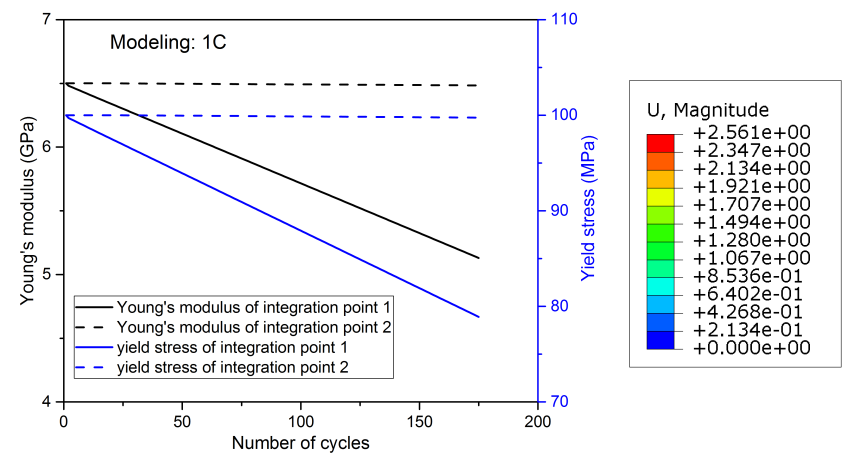

(a)

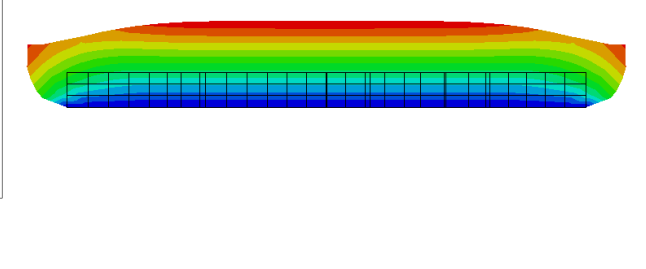

(b)

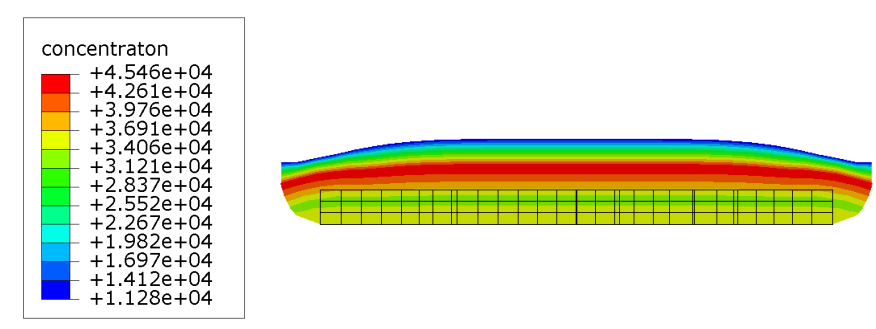

(c)

Fig 9. (a) The corresponding damage affected Young's modulus and yield strength on two integration points versus the number of cycles, (b) the cross-sectional view of deformed active layer after 150 cycles, (c) the cross-sectional view of concentration distribution within active layer after 150 cycles. 

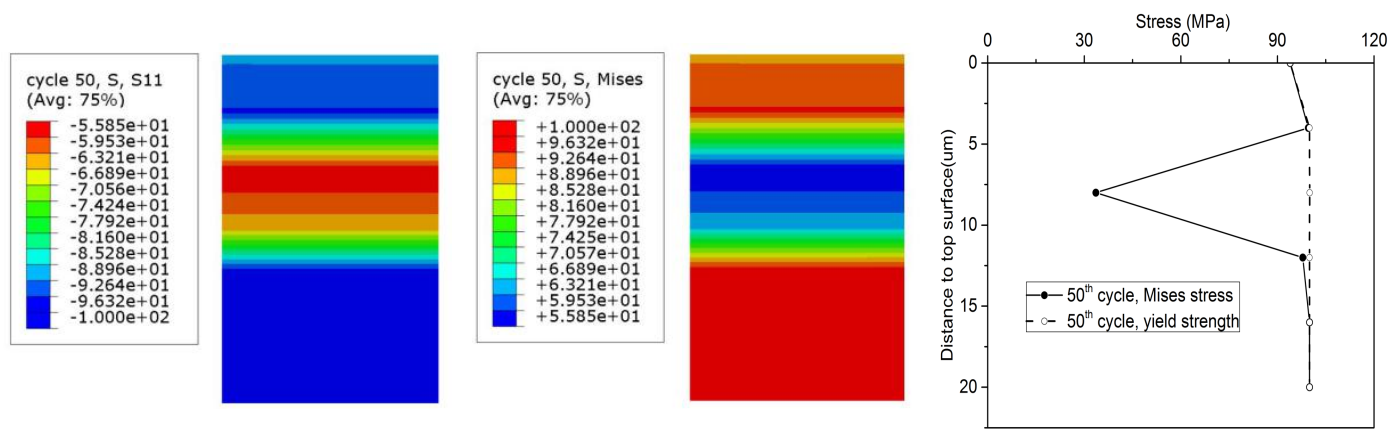

(a)
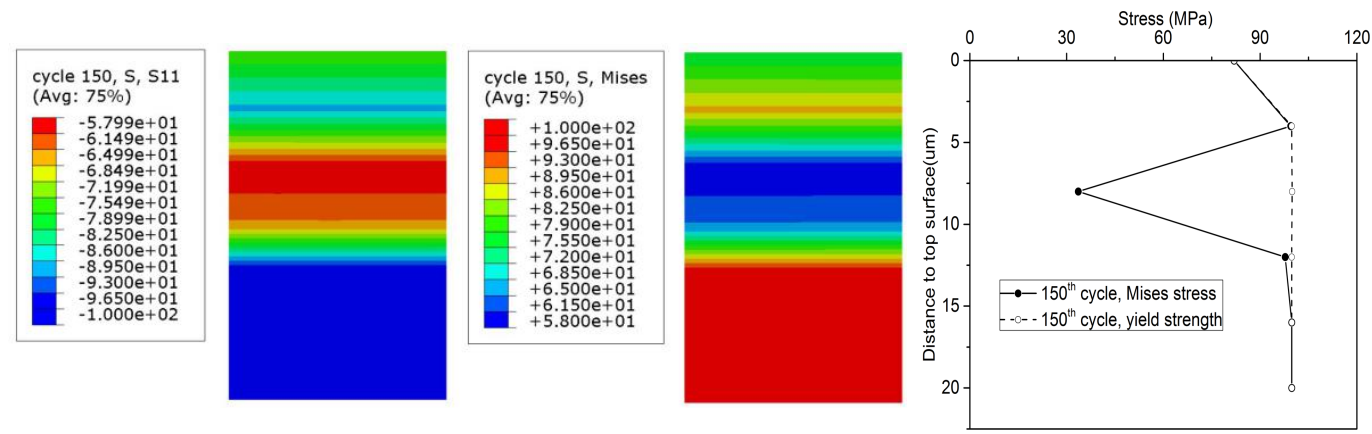

(b)

Fig 10. (a) The distribution of in-plane stress component and von Mises stress on the thickness section at the end of lithiation phase of $50^{\text {th }} \mathrm{cycle}$, (b) the distribution of in-plane stress component and von Mises stress on the thickness section at the end of lithiation phase of $150^{\text {th }}$ cycle

For the proposed fatigue damage model shown in Eq. 15, with certain material properties including fatigue ductile coefficient as well as fatigue ductile exponent, the produced damage is determined by plastic strain range of each cycles. Fig 11 (a) and (b) present the stress-strain hysteresis loops for $50^{\text {th }}$, $100^{\text {th }}$ and $150^{\text {th }}$ cycle on integration point 1 as well as integration point 2 at $1 \mathrm{C}$. The values of strain on horizontal axis are given as relative values so as to compare the change of strain at these three cycles $\left(50^{\text {th }}, 100^{\text {th }}\right.$ and $150^{\text {th }}$ cycle). Due to the fact that the elastic-perfectly plastic constitutive equation is applied in modelling the post-yielding behaviour, the stress in plastic strain generation phase keeps constant at yield. At the initial phase of lithiation, the top surface of electrode experiences a tensile stress. With the proceeding of Li-ion insertion, the stress on top surface gradually converts to a compressive stress which results in the compressive strain generated in lithiation phase. While for the delithiation course, the extraction of Li-ion shifts the compressive stress toward tensile stress for top surface and leads to the generation of tensile strain during the de-intercalation phase. On the contrary, the bottom surface bonded to current collector is subjected to compressive stress during the entire lithiation-delithiation process and compressive strain is generated at the end stage of intercalation phase.

For integration point 1 , it can be found that the yield strength of the material is gradually reduced with the proceeding of cycles due to the accumulated damage effect and the slope representing Young's modulus is also decreased. According to the Hooke's law, this synchronous decrease will lead to the generation of the same elastic strain range for these three cycles as 0.03076 . For integration point 2 , the generated elastic strain ranges for three cycles are almost the same and the tiny difference of stress ranges results in the very little difference of elastic strain ranges.

It is also observed that the plastic strain range $\Delta \varepsilon_{p}$ from three cycles are identical to 0.03875 for integration point 1 and 0.00202 for integration point 2 . In Fig 11 (c), the plastic strain range on two 
integration points versus the number of cycles are depicted. After a few initial cycles, the calculated plastic strain range per cycle is almost constant. It is because of that, according to the relevance assumption and linearly accumulated damage obtained by experiment test, the Young's modulus and yield strength are synchronously revised by accumulated damage in the numerical procedure. Hence, with certain fatigue parameters, the fatigue damage model will produce identical damage value per cycle after the initial oscillation phase, which makes the mechanical damage of electrode almost accumulate in a linear way. Besides, as shown in the previous section, the proposed cumulative damage model with different $n_{r}\left(n_{r} \neq 1\right)$ is capable of simulating the non-linear fatigue damage accumulation process based on the stress-strain response per cycle. Under this circumstances, the Young's modulus and yield strength are asynchronously changed by damage. Additionally, the stressstrain hysteresis loops present the cyclic softening characteristic for surface adhering to electrolyte. With the proceeding of cycles, the fatigue damage effect is equivalently converted to the decrease of yield strength of electrode material, which makes the resistance to deformation decrease as cycle proceeds. Due to the slower fatigue damage accumulation rate, the bottom surface of electrode plate presents the weak cyclic softening characteristic contrary to the rapid cyclic softening of top surface.

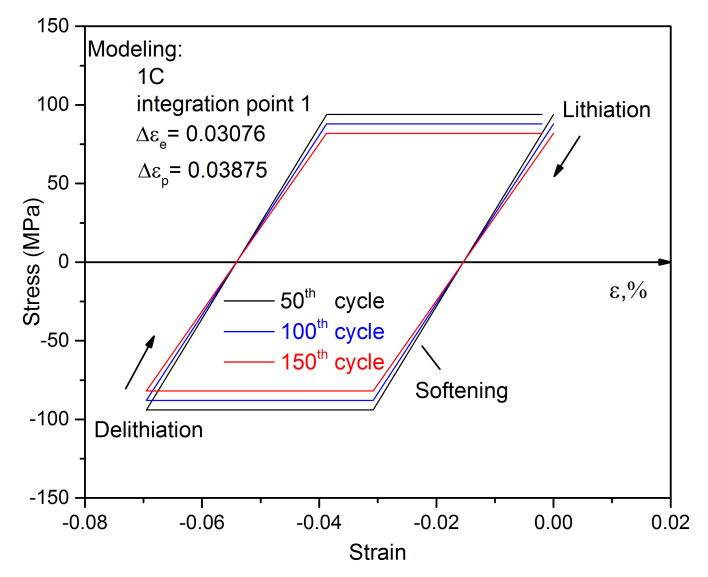

(a)

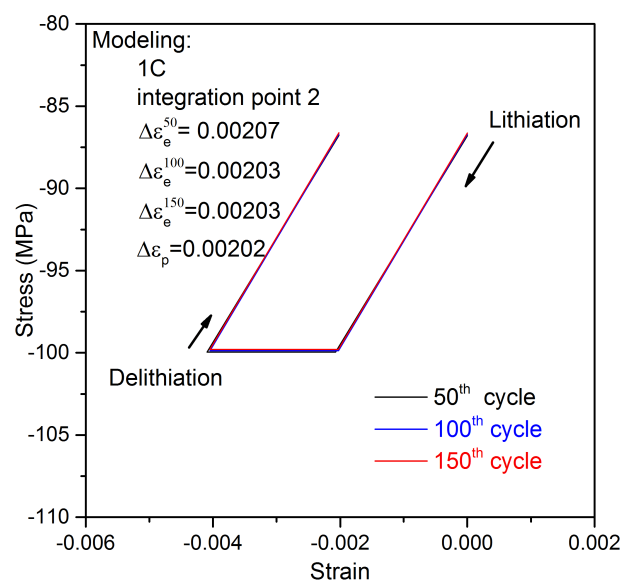

(b)

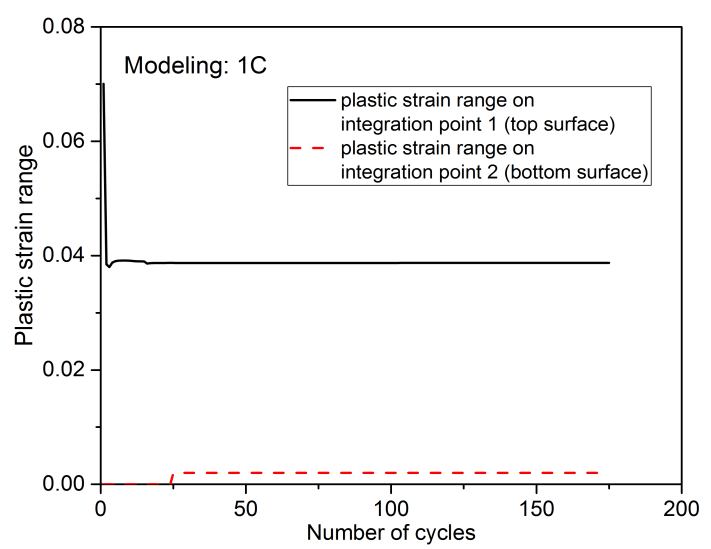

(c)

Fig 11. The stress-strain hysteresis loops for $50^{\text {th }}, 100^{\text {th }}, 150^{\text {th }}$ cycle at $1 \mathrm{C}$ on (a) integration point 1 , (b) integration point 2, (c) the calculated plastic strain range on two integration points versus the number of cycles under $1 \mathrm{C}$.

Fig 12 (a) and (b) present the stress-strain hysteresis loops within three continuous cycles at steady state under $1 \mathrm{C}$ for integration point 1 (top surface of electrode plate) and integration point 2 (bottom surface of electrode plate). The relative values of strain are shown on the horizontal axis. It is accessible to observe a shift of stress-strain curves along the negative direction of strain axis of both 
two integration points. For integration point 1 , this is due to the fact that the plastic strain generated from tensile process is less than the reverse plastic strain in compressive phase. While for integration point 2 , it is because of there is only compressive strain produced in the lithiation-delithiation process. These will cause the accumulation of plastic strain as well as the potential ratcheting collapse. The increments of plastic strain $\varepsilon_{p i}$ are roughly equal on two integration points per cycle, as -0.00199 for integration point 1 and -0.00202 for integration point 2 .

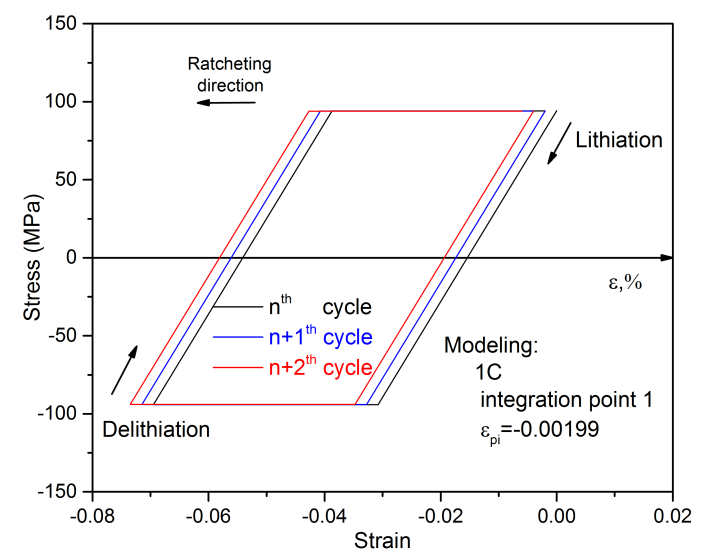

(a)

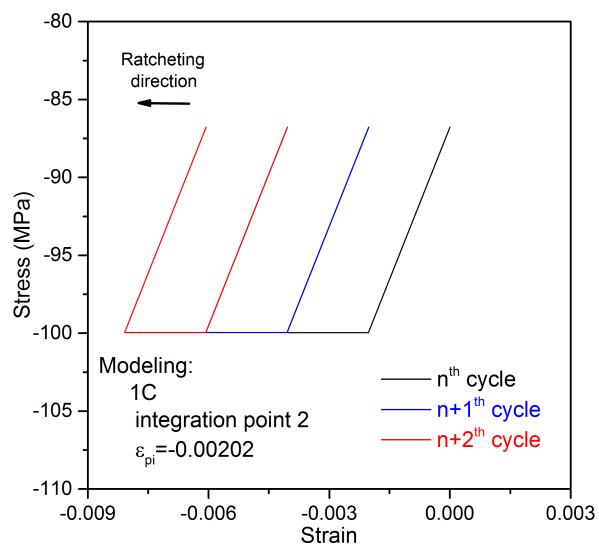

(b)

Fig 12. Three continuous stress-strain hysteresis loops at $1 \mathrm{C}$ for (a) integration point 1 , (b) integration point 2

In addition, the interaction between the formation of diffusion induced stress and diffusion behaviour is also taken into account in the established approach. Fig 13 (a) presents the variation of hydrostatic stress for two integration points within three continuous cycles under $1 \mathrm{C}$, and Fig 13 (b) shows the transiently changed diffusivity affected by hydrostatic stress based on Eq. 5 . It can be seen that the variation of diffusivity follows the same trend with the changing of hydrostatic stress. For integration point 1 on the top surface of active layer, the influence from hydrostatic stress reduces the diffusivity from the intermediate stage of intercalation process to the intermediate stage of de-intercalation phase, and magnifies it at other moments. Contrary to top surface, the hydrostatic stress always hinders the diffusion behaviour in the lithiation-delithiation course for bottom face. The maximum effects from hydrostatic stress on diffusivity reach $4.7 \%$ and $5.5 \%$ for integration point 1 and integration point 2 respectively.

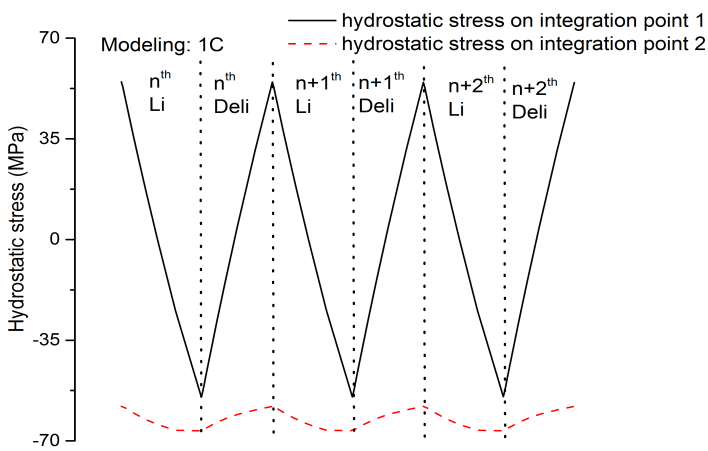

(a)

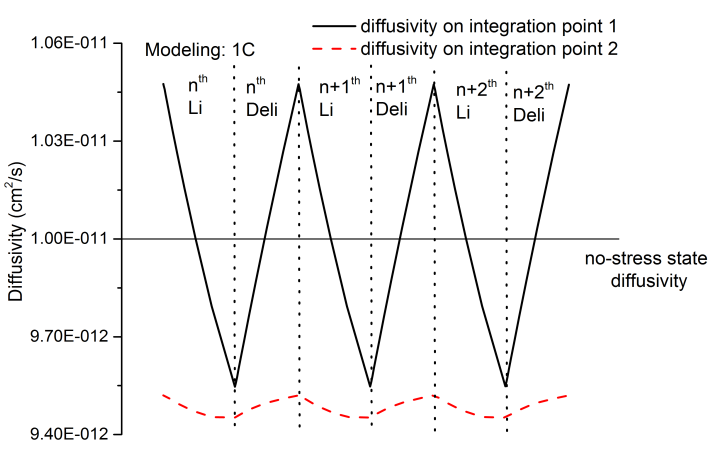

(b)

Fig 13. (a) The variation of hydrostatic stress for two integration points within three continuous cycles, (b) the transient changed diffusivity affected by diffusion induced stress within three continuous cycles 
Fig 14 (a) and (b) present the variation of plastic strain for the top surface and bottom surface of electrode within one cycle at $0.5 \mathrm{C}, 1 \mathrm{C}$ and $2 \mathrm{C}$ respectively. The solid lines and dot lines here represent the lithiation phase and delithiation phase. Under various electrochemical conditions, for top surface, both the lithiation and delithiation processes comprise an initial elastic phase and a subsequent plastic phase. It can be seen that, the magnitude of plastic strain range is co-determined by the plastic strain rate and yielding duration. By observing from Fig 14 (a) and (b), it is available to find that the plastic strain rate increases as the $\mathrm{C}$-rate rises as expected, while the yielding duration decreases with the growth of $\mathrm{C}$-rate. With certain fatigue parameters, the generated plastic strain range decides the corresponding fatigue damage for one cycle. Hence, the balance mechanism between plastic strain rate and yielding duration decides the variation of the produced fatigue damage per cycle under the performed electrochemical conditions. For the bottom surface, the intercalation phase will present yielding behaviour and de-intercalation phase is always in elastic status. The produced plastic strain range under three operation scenarios are far less than that of the top surface, which means the corresponding fatigue damage of top surface greatly exceeds damage on bottom surface and dominates the mechanical degradation of electrode under the researched electrochemical loads.

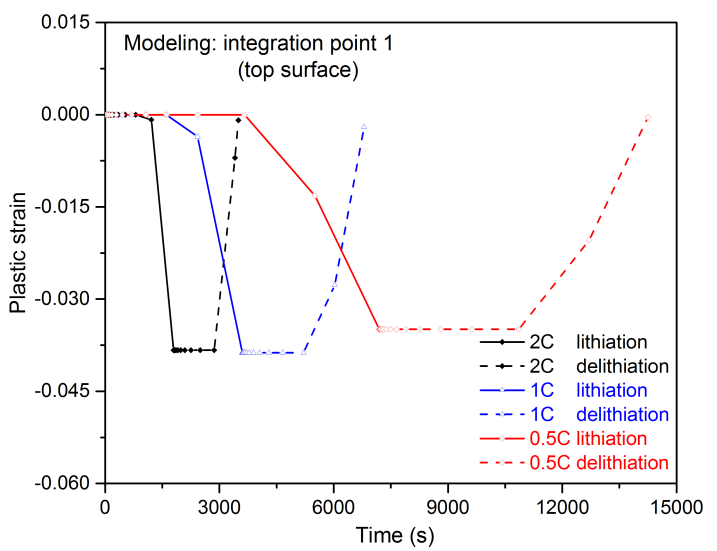

(a)

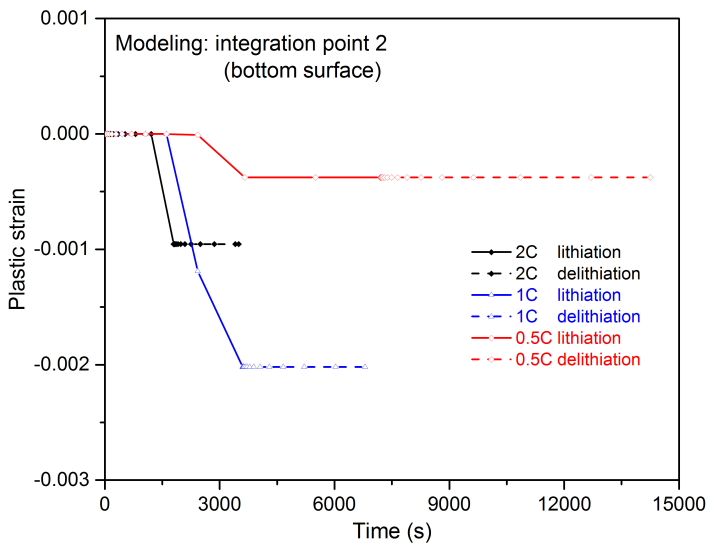

(b)

Fig 14. The variation of plastic strain within one cycle under different electrochemical loads $(0.5 \mathrm{C}$,

1C, 2C) for (a) integration point 1(top surface), (b) integration point 2(bottom surface)

In Fig 15 (a) and (b), the results of stress-strain hysteresis loops for two locations (top surface and bottom surface) under different C-rates are shown, and the results correspond to $100^{\text {th }}$ cycle for three operation strategies. As the elastic-perfectly plastic constitutive equation is employed in the numerical simulations, the corresponding stress state when plastic deformation forms, denotes the yield strength which is modified by the accumulated fatigue damage cycle by cycle. The comparison of yield stress between top surface and bottom surface presents that the top surface with lower yield stress subjects to more fatigue damage and is more prone to fracture under all operation strategies. 


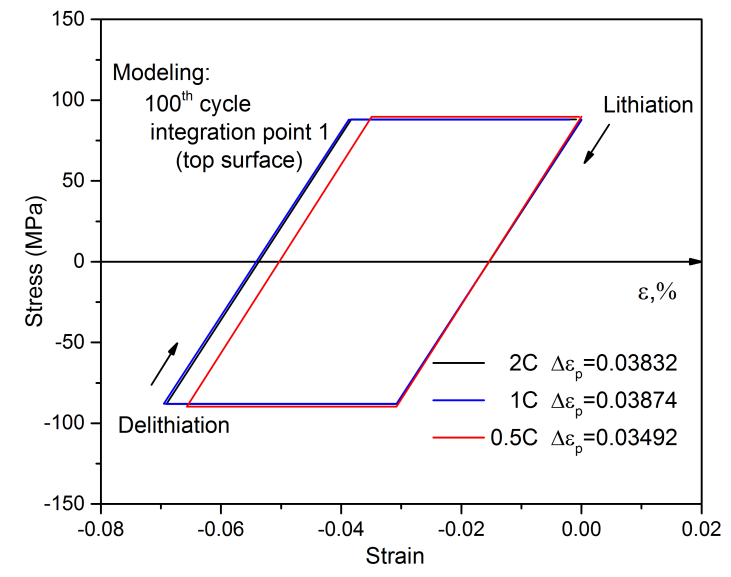

(a)

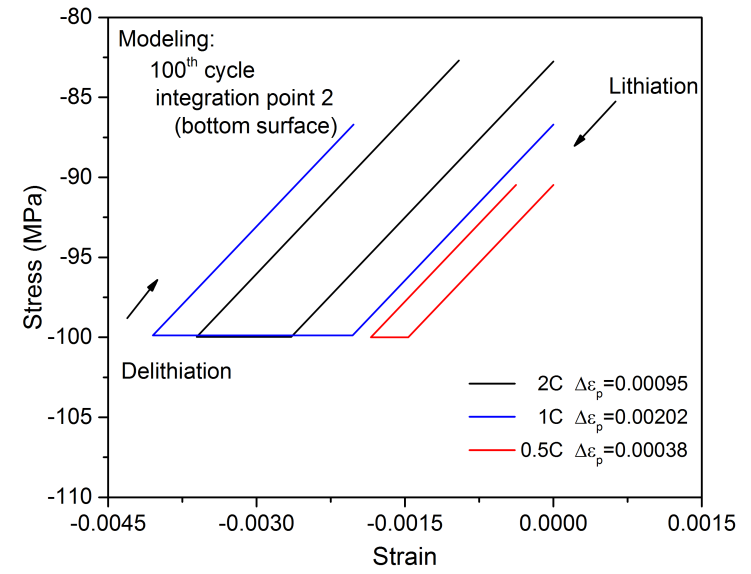

(b)

Fig 15. The stress-strain hysteresis loops at $100^{\text {th }}$ cycle under $0.5 \mathrm{C}, 1 \mathrm{C}$ and $2 \mathrm{C}$ for (a) integration point 1 (top surface), (b) integration point 2(bottom surface).

\section{Conclusions}

A fatigue damage evaluation approach to track the mechanical degradation history of battery electrode is presented in this paper. The numerical procedure is built on the theory of continuum damage mechanics and takes couple effect between diffusion and stress formation as well as the fatigue damage affected stress-strain response into account. The presented approach is implemented in ABAQUS by developing finite element subroutines and is proved to be able to evaluate multifarious types of fatigue damage accumulation trends of battery electrode. The strategy is established to correlate the electrochemical degradation and mechanical fatigue deterioration. Cyclic chargedischarge tests with respect to different electrochemical conditions are carried out to parameterize and verify the fatigue damage model as well as the aforementioned relevance assumption.

According to the number of cycles to failure, the mechanical deterioration of electrode is suggested to be a strain controlled failure. Manson-coffin fatigue model is employed to describe the damage accumulation. The relevance assumption, that electrochemical damage is identical to the mechanical damage on the top surface of layered electrode, is proposed to associate electrochemistry degradation with mechanical deterioration. Based on the aforementioned assumption, circulating charging-discharging experiment at $1 \mathrm{C}$ is conducted to fit the fatigue ductile exponent and fatigue ductile coefficient in the proposed cumulative damage model. For model validation, the parameterized damage model is applied to track the damage evolution of electrode cycled at $0.5 \mathrm{C}$ and 2C. Circulating charging-discharging experiments at $0.5 \mathrm{C}$ and $2 \mathrm{C}$ are performed and compared with numerical simulations to validate the applicability of the parameterized damage model and relevance assumption.

Results show that the damage of electrode is layered distributed along the direction of Li-ion flow and the electrode surface adhering to electrolyte is more prone to fracture. Additionally, the fatigue damage in this work is a function of plastic strain range which is co-determined by the plastic strain rate and the duration of yielding. The increasing of $\mathrm{C}$-rate will lead to the rising of plastic strain rate as well as the falling of yielding time. The present research work suggests that it is accessible to employ the continuum damage approach to track the mechanical degradation of electrode cycled under electrochemical conditions. 


\section{Acknowledgments}

The authors gratefully acknowledge the supports from the China Scholarship Council, Shanghai Automobile Industry Science and Technology Development Foundation (1801), National Natural Science Foundation of China (51828501), the Higher Education Discipline Innovation Project (111 Project) under the funding code B13020, University of Strathclyde and East China University of Science and Technology during the course of this work.

\section{Appendix A.}

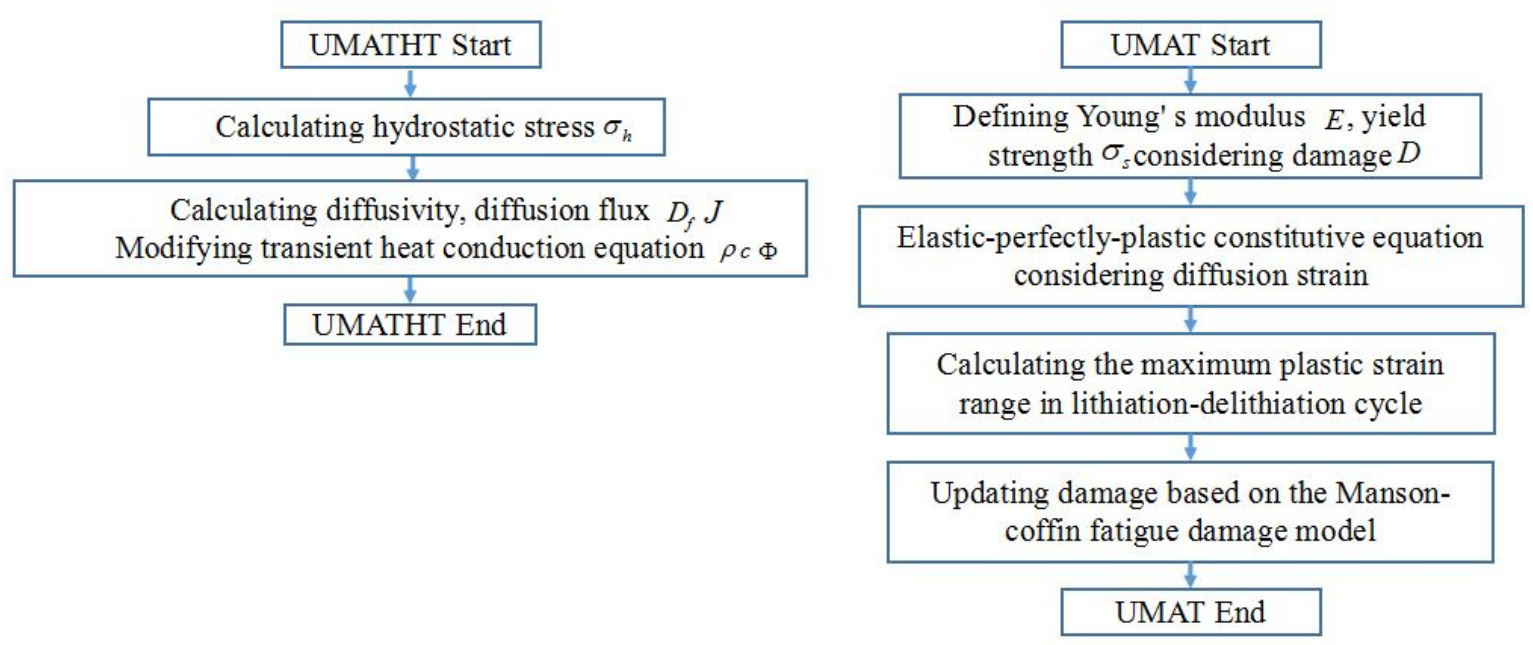

Fig A1. The flow chat of UMAT-UMATHT subroutines

\section{References}

[1] V. Etacheri, R. Marom, R. Elazari, G. Salitra, D. Aurbach. Challenges in the development of advanced Li-ion batteries: a review. Energy Environmental Science, 2011, 4, 3243.

[2] M.A. Hannan, M.S.H. Lipu, A. Hussain, A. Mohamed. A review of lithium-ion battery state of charge estimation and management system in electric vehicle applications: Challenges and recommendations. Renewable and Sustainable Energy Reviews, 2017, 78: 834-854.

[3] C. Xu, L. Wen, L. Ji, J. Zhou. An analytical model for the fracture behavior of the flexible lithium-ion batteries under bending deformation. European Journal of Mechanics - A/Solids, 2019, 73: 47-56.

[4] A. Mukhopadhyay, B.W. Sheldon. Deformation and stress in electrode materials for Li-ion batteries. Progress in Materials Science, 2014, 63, 58-116.

[5] H. Wu, Z. Xie, Y. Wang, C. Lu, Z. Ma. Modeling diffusion-induced stress on two-phase lithiation in lithium-ion batteries. European Journal of Mechanics - A/Solids, 2018, 71: 320-325.

[6] R. Deshpande, Y. Cheng, M. Verbrugge. Modeling diffusion-induced stress in nanowire electrode structures. Journal of Power Sources, 2010, 195(15): 5081-5088.

[7] L. Weng, J. Zhou, R. Cai. Analytical model of Li-ion diffusion-induced stress in nanowire and negative Poisson's ratio electrode under different operations. International Journal of Mechanical Sciences, 2018, 141: 245-261. 
[8] L. Ji, Z. Guo. Analytical modeling and simulation of porous electrodes: Li-ion distribution and diffusion-induced stress. Acta Mechanica Sinica, 2018, 34: 187-198.

[9] X.C. Zhang, W. Shyy, A.M. Sastry. Numerical simulation of intercalation-induced stress in Li-ion battery electrode particles. Journal of The Electrochemical Society, 2007, 154(10), A910-A916.

[10] Z. Cui, F. Gao, J. Qu. A finite deformation stress-dependent chemical potential and its applications to lithium ion batteries. Journal of the Mechanics and Physics of Solids, 2012, 60(7):1280-1295.

[11] L. Wu, X. Xiao, Y. Wen, J. Zhang. Three-dimensional finite element study on stress generation in synchrotron $\mathrm{X}$-ray tomography reconstructed nickel-manganese-cobalt based half cell. J Power Sources 2016, 336:8-18.

[12] J.Q. Zhang, B. Lu, Y.C. Song, X. Ji. Diffusion induced stress in layered Li-ion battery electrode plates. Journal of Power Sources, 2012, 209, 220-227.

[13] M. Liu. Finite element analysis of lithium insertion-induced expansion of a silicon thin film on a rigid substrate under potentiostatic operation. Journal of Power Sources, 2015, 275, 760-768.

[14] R. Xu, K. Zhao. Electrochemomechanics of Electrodes in Li-lon Batteries: A Review. Journal of Electrochemical Energy Conversion and Storage, 2016, 13(3): 030803

[15] Y Hu, X Zhao, Z Suo. Averting cracks caused by insertion reaction in lithium-ion batteries. Journal of Materials Research, 2010, 25(06): 1007-1010.

[16] Chew H, Hou B, Wang X, Xia S. Cracking mechanisms in lithiated silicon thin film electrodes. International Journal of Solids and Structures, 2014, 51(23-24): 4176-4187.

[17] M. Klinsmann, D. Rosato, M. Kamlah, R.M. McMeeking. Modeling crack growth during Li insertion in storage particles using a fracture phase field approach. Journal of the Mechanics and Physics of Solids, 2016, 92:313-344.

[18] M. Klinsmann, D. Rosato, M. Kamlah, R.M. McMeeking. Modeling crack growth during Li extraction in storage particles using a fracture phase field approach. Journal of the Electrochemical Society, 2016, 163:102-118.

[19] G. Sun, T. Sui, B. Song, H. Zheng, L. Lu, A.M. Korsunsky. On the fragmentation of active material secondary particles in lithium ion battery cathodes induced by charge cycling. Extreme Mechanics Letters, 2016, 9(3): 449-458.

[20] X. Hu, Y. Zhao, R. Cai, J. Zhou. Surface effected fracture behavior of nano-spherical electrodes during lithiation reaction. Materials Science and Engineering: A, 2017, 707: 92-100.

[21] J. Li, A.K. Dozier, Y. Li, F. Yang, Y.-T. Cheng. Crack pattern formation in thin film lithium-ion battery electrodes. Journal of The Electrochemical Society, 2011, 158(6): A689-A694.

[22] L. Yang, H. Chen, H. Jiang, W. Song, D. Fang. Lithium redistribution around the crack tip of lithiumion battery electrodes. Scripta Materialia, 2019, 167: 11-15.

[23] X.C. Zhu, H.F. Chen, W.L. Luan. The diffusion induced stress and cracking behaviour of primary particle for Li-ion battery electrode. International Journal of Mechanical Sciences, 2020, 178: 105608.

[24] D. Chen, D. Kramer, R. Mönig. Chemomechanical fatigue of LiMn1.95Al0.0504 electrodes for lithium-ion batteries. Electrochimica Acta, 2018, 259: 939-948. 
[25] M.J. Mühlbauer, O. Dolotko, M. Hofmann, H. Ehrenberg, A. Senyshyn. Effect of fatigue/ageing on the lithium distribution in cylinder-type Li-ion batteries. Journal of Power Sources, 2017, 348: 145-149.

[26] S. Schweidler, L. de Biasi, G. Garcia, A. Mazilkin, P. Hartmann, T. Brezesinski. J. Janek. Investigation into mechanical degradation and fatigue of High-Ni NCM cathode material: a long-term cycling study of full cells. Applied Energy Materials, 2019, 2: 7375-7384.

[27] D. Sørensen, M. Heere, J. Zhu, M. Darma, S. Zimnik, M. Mühlbauer, L. Mereacre, V. Baran, A. Senyshyn, M. Knapp, H. Ehrenberg. Fatigue in high-energy commercial Li batteries while cycling at standard conditions: an in situ neutron powder diffraction study. Applied Energy Materials, 2020, 3: 6611-6622.

[28] L.M. Kachanov. On time to rupture in creep conditions. Izviestia Akademii Nauk SSSR, Otdelenie Tekhnicheskikh Nauk, 1958, 8: 26-31.

[29] S. Murakami. Continuum damage mechanics: a continuum mechanics approach to the analysis of damage and fracture. Springer, Dordrecht, Heidelberg, London, New York (2012)

[30] J.L. Chaboche. Continuum damage mechanics: Part I-general concepts. Journal of Applied Mechanics, 1988, 55(1), 59-64.

[31] J.L. Chaboche. Continuum damage mechanics: Part II—damage growth, crack initiation, and crack growth. Journal of Applied Mechanics, 1988, 55(1), 65-72.

[32] N. Liu, X. Cui, J. Xiao, J. Lua, N. Phan. A simplified continuum damage mechanics based modeling strategy for cumulative fatigue damage assessment of metallic bolted joints. International Journal of Fatigue, 2020, 131: 105302.

[33] Z. Zhan, Q. Meng, W. Hu, Y. Sun, F. Shen, Y. Zhang. Continuum damage mechanics based approach to study the effects of the scarf angle, surface friction and clamping force over the fatigue life of scarf bolted joints. International Journal of Fatigue, 2017, 102: 59-78.

[34] R. Grantab, V.B. Shenoy. Location- and orientation-dependent progressive crack propagation in cylindrical graphite electrode particles. Journal of The Electrochemical Society, 2011, 158(8):A948

[35] S.L. Zhang. Chemomechanical modeling of lithiation-induced failure in high-volume-change electrode materials for lithium ion batteries. Computational Materials, 2017, 3:7

[36] H. Hibbitt, B. Karlsson, P. Sorensen, 2012. ABAQUS Theory Manual, Version 6.12. Pawtucket, Rhode Island, USA.

[37] S.S. Manson. Fatigue: A complex subject some simple approximations. Journal of Experiment Mechanics, 1965, 5(7):193-226.

[38] S. Baltic, J. Magnien, H. Gänser, T. Antretter, R.Hammer. Coupled damage variable based on fracture locus: Modelling and calibration. International Journal of Plasticity, 2020, 126: 102623

[39] J. Zhu, X. Zhang, H. Luo, E. Sahraei. Investigation of the deformation mechanisms of lithium-ion battery components using in-situ micro tests. Applied Energy, 2018, 224: 251-266.

[40] R. Xu, H. Sun, L. Vasconcelos, K. Zhao. Mechanical and structural degradation of LiNixMnyCozO2 cathode in Li-ion Batteries: An Experimental study. Journal of Electrochemical Society, 2017, 164(13):A3333-A3341 
[41] A. Verma, K. Smith, S. Santhanagopalan, D. Abraham. K. Yao, P. Mukherjee. Galvanostatic intermittent titration and performance based analysis of LiNi0.5Co0.2Mn0.3O2 cathode. Journal of The Electrochemical Society, 2017, 164(13):A3380-A3392

[42] O. Dolotko, A. Senyshyn, M.J. Muhlbauer, K. Nikolowski, H. Ehrenberg. Understanding structural changes in NMC Li-ion cells by in situ neutron diffraction. Journal of Power Sources, 2014, 255:197203

[43] A. Sarkar, P. Shrotriya, A. Chandra. Simulation-driven selection of electrode materials based on mechanical performance for Lithium-Ion battery. Materials, 2019, 12, 831.

[44] R. Xu, L. Vasconcelos, K. Zhao. Computational analysis of chemomechanical behaviors of composite electrodes in Li-ion batteries. Journal of Materials Research, 2016, 31(18), 2715-2727.

[45] Y. Shi, M. Zhang, C. Fang, Y. S. Meng, Urea-based hydrothermal synthesis of LiNi0.5Co0.2Mn0.3O2 cathode material for Li-ion battery. Journal of Power Sources, 2018, 394: 14-121.

[46] X. Jia, M. Yan, Z. Zhou, X. Chen, C. Yao, D. Li, D. Chen, Y. Chen. Nd-doped LiNi0.5Co0.2Mn0.3O2 as a cathode material for better rate capability in high voltage cycling of Li-ion batteries. Electrochimica Acta, 2017, $254: 50-58$.

[47] Q. Liu, Y. Liu, F. Yang, H. He, X. Xiao, Y. Ren, W. Lu, E. Stach, J. Xie. Capacity fading mechanism of the commercial 18650 LiFePO4-based Lithium-ion batteries: an in situ time-resolved high-energy synchrotron XRD study. Applied Materials \& Interfaces, 2018, 10: 4622-4629.

[48] G. Zheng, Q. Zhang, J. Cha, Y. Yang, W. Li, Z. Seh, Y. Cui. Amphiphilic surface modification of hollow carbon nanofibers for improved cycle life of lithium sulfur batteries. Nano Letters, 2013, 13: 12651270 .

[49] A. Yasmin, M. Shehzad, X. Ding, J. Wang, R. Yu, M. Deng, Z. Tang, C. Chen. A first report on ex-situ synthesis and utilization of pure La4NiLiO8 in emerging highperformance safe batteries. Journal of Alloys and Compounds, 2020, 821: 153208

[50] X. Jia, M. Yan, Z. Zhou, X. Chen, C. Yao, D. Li, D. Chen, Y. Chen. Nd-doped LiNi0.5Co0.2Mn0.3O2 as a cathode material for better rate capability in high voltage cycling of Li-ion batteries. Electrochimica Acta, 2017, 254: 50-58.

[51] J. Gilbert, I. Shkrob, D. Abraham. Transition metal dissolution, Ion migration, electrocatalytic reduction and capacity loss in Lithium-Ion full cells. Journal of The Electrochemical Society, 2017, 164: A389-A399 